linkage $)=-3.36 \times 10^{-6}$, for this purpose. From these assumptions, the magnetic anistropy $\Delta K=$ $K_{3}-K_{1}$ was found to be $-615.9 \times 10^{-6}$ for ovalene. This is a very high value. The contribution of each $\pi$-electron to the magnetic anisotropy can be expressed, conventionally, by the classical theory, $\Delta K=-\left(N e^{2} / 4 m c^{2}\right) \Sigma \bar{r}^{2}$, where $\vec{r}$ is the mean radius

of a circular orbit of a $\pi$-electron. For ovalene, $r$ is calculated to be $2 \cdot 12 \mathrm{~A}$. This is also a high value, when it is remembered that $\bar{r}$ is $1.44 \mathrm{~A}$. for benzene, 1.57 A. for naphthalene, 1.63 A. for anthracene and $1.86 \mathrm{~A}$. for pyrene, where these values were calculated by the same method as for ovalene from specific susceptibility data in the literature, which do not differ seriously from the values obtained with single crystals 4 .

Hidieo Akamatu Hiroo INOKUCHI

Department of Chemistry, TAKASHI HANDA

Faculty of Science,

University of Tokyo.

$$
\text { May } 1 .
$$

${ }^{1}$ Clar, E., Nature, 161, 238 (1948). Clar, E., and Frömmel, H., Chem. Ber., 82, 46 (1949).

${ }^{2}$ A kamatu, H., and Inokuchi, H., J. Chem. Phys., 18, 810 (1950).

${ }^{3}$ Shiba, H., and Hazato, G., Bull. Chem. Soc. Japan, 22,92 (1949).

'Lonsdale, K., Proc. Roy Soc., A, 159, 149 (1937).

\section{Physical Basis of Internal Calorimetry}

THE technique of 'internal calorimetry', a development of Gibbs's 'heated thermocouple' method', was devised for the measurement of blood flow in living tissues. The instrument consists of a small constantan filament connected to a source of electrical potential, variable at will. An attached thermocouple records the filament temperature. The energy required to maintain thermal equilibrium with the instrument embedded in the experimental material is recorded electrically. Its relation to blood flow has already been described ${ }^{2}$; our present concern, however, is with the non-circulatory heat losses from the filament.

With a recorder embedded in a gel- 5 per cent gelatine in water-and heated $1^{\circ} \mathrm{C}$. above ambient temperature, temperatures were measured thermoelectrically along a line perpendicular to the filament and also back along the leads. Temperatures were less than $0.01^{\circ} \mathrm{C}$. in excess of the ambient temperature at a point in the gel $5 \mathrm{~mm}$. from the filament and at a point on the wire distant $1 \mathrm{~cm}$. from the filament.

It can be shown mathematically ${ }^{3}$ that, with an electrically heated spherical source of heat in an infinite mass of material, a steady state is approached in which the relation between heat production and heat loss is represented by the equation :

$$
I^{2} R=4 K \cdot \pi \cdot r \theta
$$

(where $K$ is thermal conductivity of the substance, $r$ is radius of sphere, $\theta$ is temperature elevation of sphere, $I$ is heating current and $R$ is heating filament resistance). Where $\theta, r$ and $R$ are fixed, $I^{2} / K$ is thus constant.

That a similar relation may be applied to the present work was demonstrated using a standard instrument to record in air, chloroform, 'Vaseline', glycerine, water and mercury. Temperature was carefully controlled and convection errors eliminated by methods similar to those of Read and Lloyd ${ }^{4}$. In each case, the value of $I^{2}$ for $\theta=I^{\circ} \mathrm{C}$. was determined. Taking the values of $K$ as given in the "International Critical Tables", the value of $I^{2} / K$ was obtained for each substance and found to be virtually constant at 14.0 $0 \times 10^{4}( \pm 1$ per cent). This ceased to be true if the depth of insertion or the bulk of fluid was not sufficient. The limits determined in 5 per cent gelatine, however, were $1 \mathrm{~cm}$. depth of insertion and $5 \mathrm{~mm}$. of substance surrounding the thermocouple.

It was thus shown, under these experimental conditions, that with 0 constant, $I^{2} R \propto K$. In further experiments where $K$ was constant, the relation between $\theta$ and $I^{2} R$ was investigated and found to be linear except when $\theta$ was so large that the limiting conditions were exceeded.

These findings facilitate the standardization of blood-flow recorders in a manner to be described elsewhere. Moreover, they show that the method of 'internal calorimetry' may be used as a simple, quick means of measuring thermal conductivities, particularly in animal tissues.

\section{W. Chester}

J. GRAYSON

Department of Applied Mathematics, and

Department of Physiology, University of Bristol.

May 10.

${ }^{1}$ Gibbs, F. A., Proc. Soc. Exp. Biol., N.Y., 31, 141 (1933).

${ }^{2}$ Grayson, J., J. Physiol., [114, 29P (1951)].

3 Carslaw, H. S. "The Mathematical Theory of the Conduction of Heat in Solids" (London: Macmillan and Co., Ltd., 1921).

${ }^{4}$ Read, J. H., and Lioyd, D. M. G., Trans. Farad. Soc., 44, 721 (1948).

\section{Effect of Tension on Water Absorption by Hair}

Ir seems to be generally assumed that the application of tensile stress to a textile fibre should reduce the amount of absorbed water in equilibrium with water vapour in the atmosphere at a given value of the relative humidity ${ }^{1}$ 'This assumption would appear to be substantiated by the recent work of White and $\mathrm{Stam}^{2}$ on human hair, in which a substantially reversible reduction of water content amounting to 50 per cent was found to occur on the application of a tensile stress of approximately $120 \mathrm{kgm} . / \mathrm{cm} .{ }^{2}$.

On the other hand, for vulcanized rubber swollen in organic solvents, the equilibrium solvent concentration is increased by tensile stresses and reduced by compressive stresses ${ }^{3}$. This result is well established experimentally, and is in quantitative agreement with the statistical theory of the swollen molecular network ${ }^{4,5}$.

In view of the opposite nature of these reported effects for fibres and for rubber, for which no immediate explanation is apparent, it seemed worth while to re-examine the question of the absorption of water by fibres under stress.

A general theory of the phenomenon may be derived by a simple extension of the usual thermodynamic treatment of swelling pressures. From such a theory Barkas ${ }^{6}$ has obtained a relation which, in the case of a simple tensile stress, may be written :

$$
s_{X} d X=-v d p,
$$

where $s_{X}$ is the fractional increment of length in the direction of the stress $X$ due to the absorption of $1 \mathrm{gm}$. of liquid, and $v$ is the specific volume of the 